Supporting Information

\title{
Graphene Oxide-Supported Three-Dimensional Cobalt-Nickel Bimetallic Sponge-Mediated Peroxymonosulfate Activation for Phenol Degradation
}

Xinyue Liu ${ }^{1}$, Haoran $\mathrm{Yu}^{1}$, Jiahui $\mathrm{Ji}^{1}$, Zhuan Chen ${ }^{1}$, Maoxi Ran ${ }^{1,3}$, Jinlong Zhang ${ }^{1,2,3}$, and Mingyang $\operatorname{Xing}^{* 1,2,3}$

${ }^{1}$ Key Laboratory for Advanced Materials and Institute of Fine Chemicals, School of Chemistry \& Molecular Engineering, East China University of Science and Technology, 130 Meilong Road, Shanghai 200237, P.R. China.

${ }^{2}$ Shanghai Engineering Research Center for Multi-media Environmental Catalysis and Resource Utilization, East China University of Science and Technology, 130 Meilong Road, Shanghai 200237, P.R. China.

${ }^{3}$ National Engineering Laboratory for Industrial Wastewater Treatment, School of Resources and Environmental Engineering, East China University of Science and Technology, 130 Meilong Road, Shanghai 200237, PR China 


\section{Table of Contents}

1. Experimental Section

2. Table S1. Proportion of C, Co, Ni and O in SCNGs

3. Figure S1. Picture of the catalysts

4. Figure S2. The kinetic rates of catalysts

5. Figure S3. Degradation of $20.0 \mathrm{mg} / \mathrm{L}$ phenol with different SCNGs

6. Figure S4. The picture of the catalyst of SCNG after the cycle experiment

7. Figure S5. Degradation of $20.0 \mathrm{mg} / \mathrm{L}$ phenol when chloride ion existed

8. Figure S6. LC-MS spectra of 4-[2-imino-pyrimidine-1(2H)-yl]aniline

9. Figure S7. LC-MS spectra of sulfanilamide 


\section{Experimental Section}

\subsection{General information.}

All chemicals, phenol (Shanghai Aladdin Reagent Co., Ltd., AR), sulfadiazine (Shanghai Aladdin Reagent Co., Ltd., AR), tetracycline hydrochloride (Shanghai Maclean Biochemical Technology Co., Ltd., AR), p-chlorophenol (Shanghai Aladdin Reagent Co., Ltd., AR), potassium persulfate (Shanghai Maclean Biochemical Technology Co., Ltd., AR), nickel nitrate hexahydrate (Adamas-beta, Shanghai Titan Chemical Technology Co., Ltd., AR), cobalt nitrate hexahydrate (Shanghai Maclean Biochemical Technology Co., Ltd., AR), hexamethine tetramine (Shanghai Aladdin Reagent Co., Ltd., AR), 2,2,6,6-tetramethylpiperidine (Shanghai Aladdin Reagent Co., Ltd., AR), 5,5-dimethyl-1-pyrrolidine-N-oxide (Shanghai Aladdin Reagent Co., Ltd., AR), p-benzoquinone (Shanghai Maclean Biochemical Technology Co., Ltd., AR), sodium hydroxide (Shanghai Lingfeng Chemical Reagent Co., Ltd., AR), sulfuric acid (Sinopharm Co., Ltd., AR) were used without further purification. Ultra-pure water was prepared with OKP-S040 Standard ultrapure water system and used in all experiments.

\subsection{Characterization.}

Electron paramagnetic resonance (EPR, instrument model: Varian E-112) with 5, 5-Dimethyl-1-Pyrrolidine-N-oxide (DMPO) and 2, 2, 6, 6-Tetramethylplperldine (TEMP) as spin-trapping reagent was used to detect the radical in the reaction under the condition as follow: a center field of $352.0 \mathrm{mT}$, a microwave frequency of $9.884 \mathrm{GHz}$, a microwave power of $6.407 \mathrm{~mW}$, and a temperature of $300.0 \mathrm{~K} . \mathrm{pH}$ meter (INESA PHS-3C) was used to measure the $\mathrm{pH}$ during the reaction. High performance liquid chromatograph (HPLC, INESA) equipped with a diode array detector and a LC-08 C18 column $(4.6 \mathrm{~mm} \times 250 \mathrm{~mm}$, ANPEL, Shanghai Anpel Experimental 
Technology Co., Ltd) was employed to analyze the concentration of pollutants. Phenol, 4-chlorophenol were detected using a mobile phase of $60 \%(\mathrm{v} / \mathrm{v})$ acetonitrile aqueous solution with detection wavelength at $272 \mathrm{~nm}$ and $264 \mathrm{~nm}$. Sulfadiazine and tetracycline were detected using a mobile phase of $20 \%$ acetonitrile and $0.2 \%$ formic acid aqueous solution with detection wavelength at $264 \mathrm{~nm}$. The molecular mass of the intermediates was tested by liquid chromatography-mass spectrometry (LC-MS, instrument model: *Q-Exactive plus). 
Table S1. The proportion of C, Co, Ni and O in SCNGs according to the results of inductively X-ray photoelectron spectroscopy (XPS).

\begin{tabular}{lllll}
\hline & $\mathrm{C}(\%)$ & $\mathrm{Co}(\%)$ & $\mathrm{Ni}(\%)$ & $\mathrm{O}(\%)$ \\
\hline Before recycling & 74.66 & 1.47 & 1.84 & 22.03 \\
\hline After recycling & 54.42 & 4.63 & 5.11 & 35.84 \\
\hline
\end{tabular}




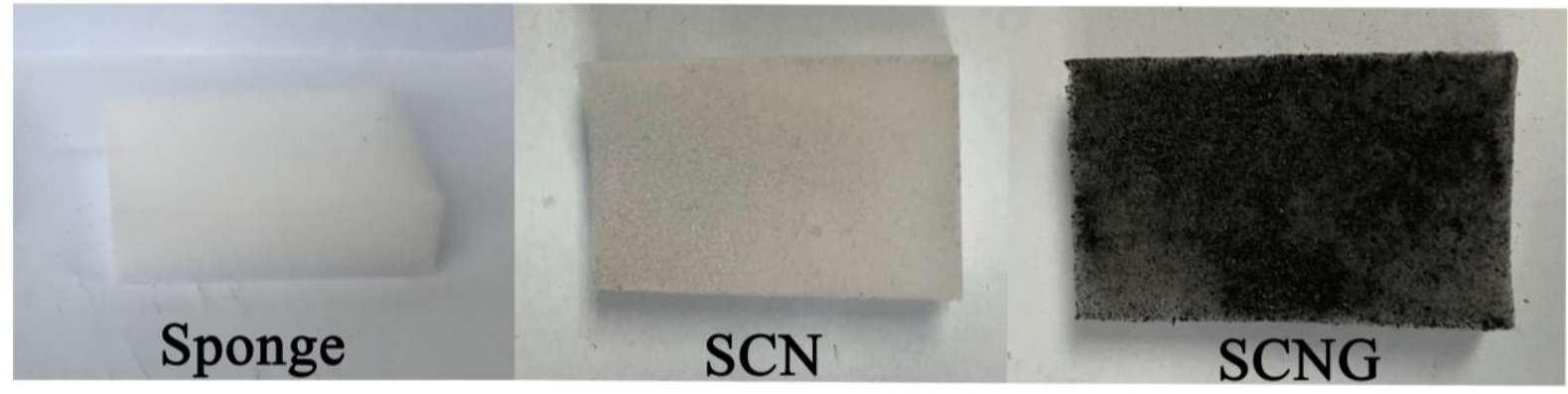

Figure S1. The picture of the catalysts of sponge, SCN and SCNG. 


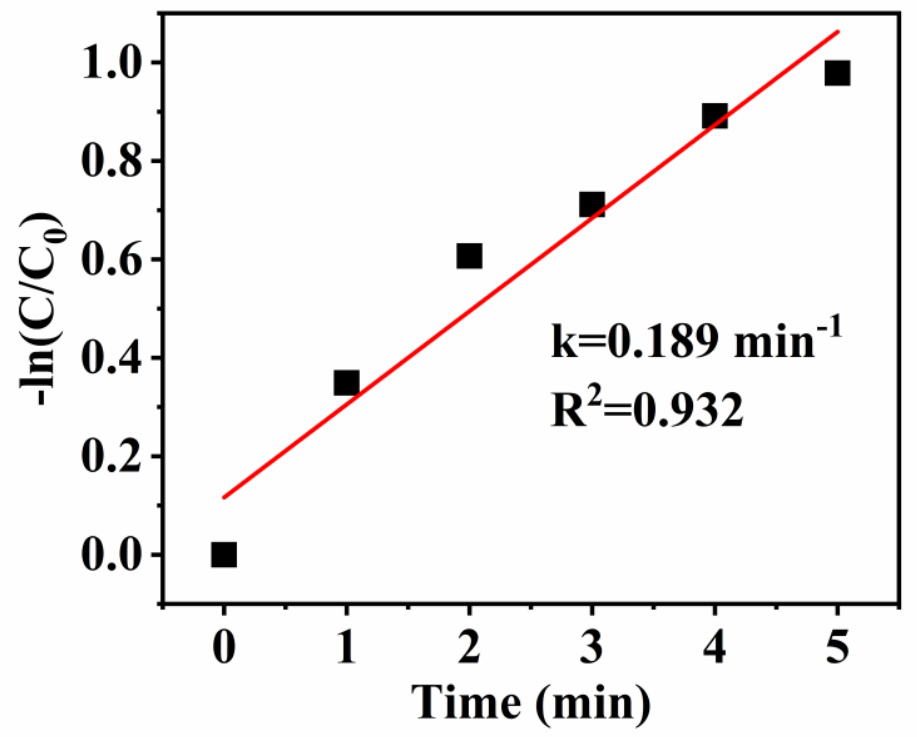

Figure S2. The kinetic rates of phenol degradation with PMS on SCNGs catalysts under optimal conditions. (Conditions: $100 \mathrm{~mL}$ system, $0.25 \mathrm{mmol} / \mathrm{L}$ PMS, three pieces of SCNGs, initial pH 7.0) 


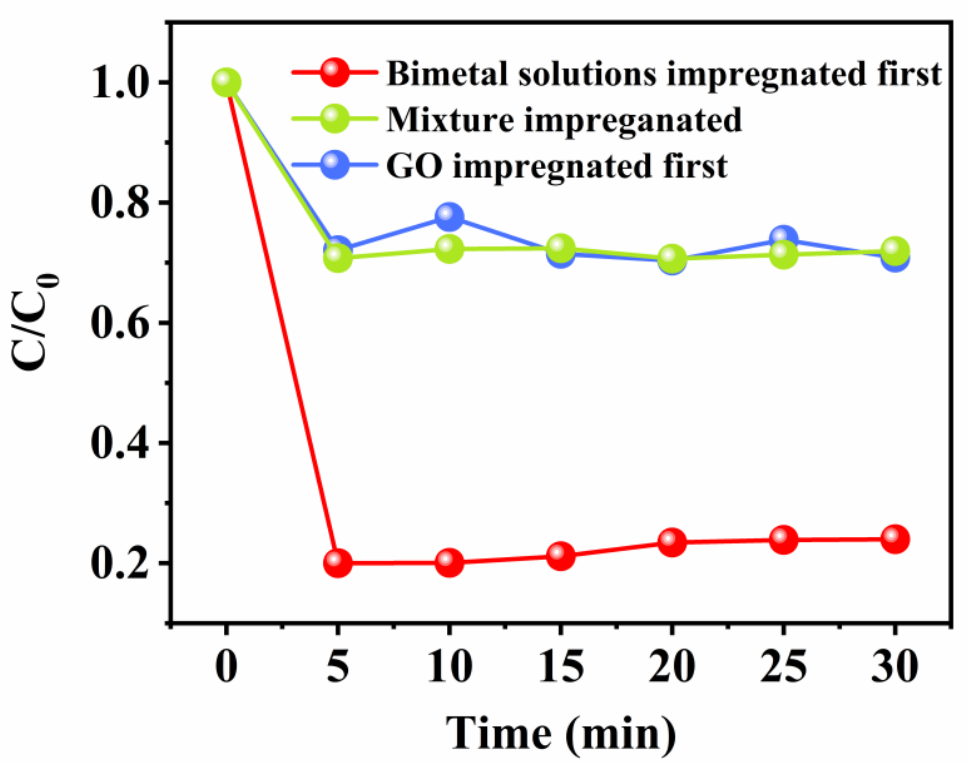

Figure S3. Degradation of $20.0 \mathrm{mg} / \mathrm{L}$ phenol with SCNGs synthesized by different procedures. 


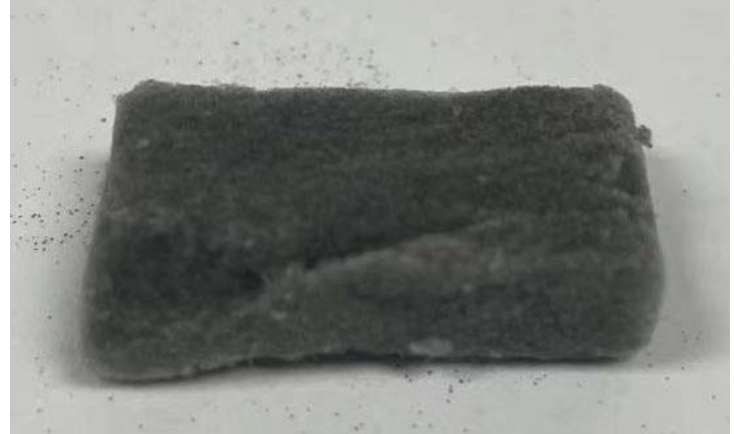

Figure S4. The picture of the catalyst of SCNG after the cycle experiment. 


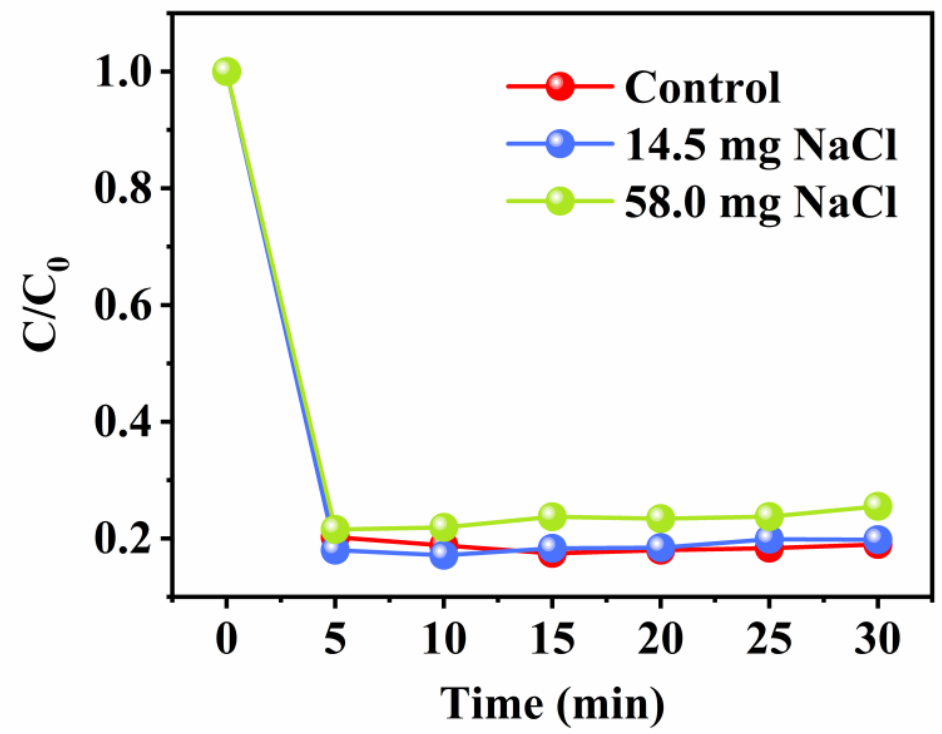

Figure S5. Degradation of $20.0 \mathrm{mg} / \mathrm{L}$ phenol when chloride ion existed. 


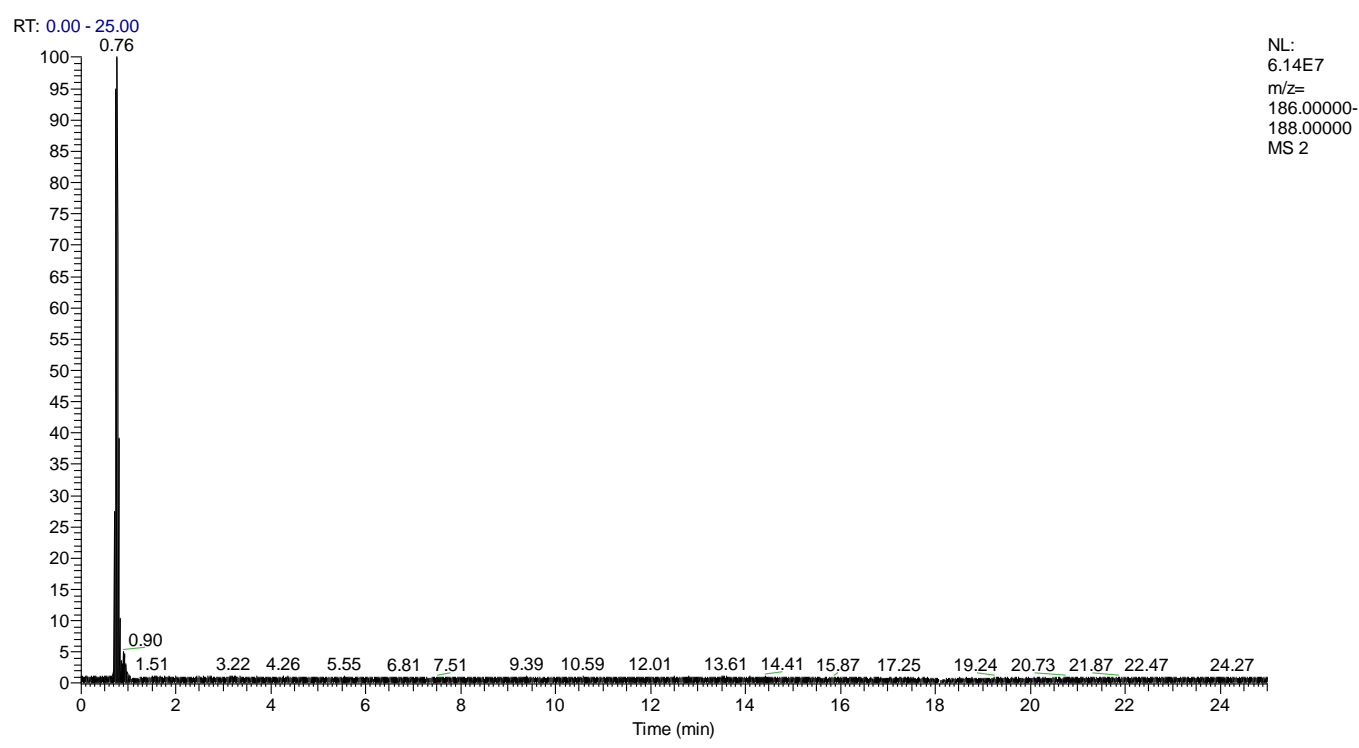

2 \#371 RT: 0.74 AV: 1 NL: $2.78 E 8$

T: FTMS + p ESI Full ms [100.0000-1000.0000]

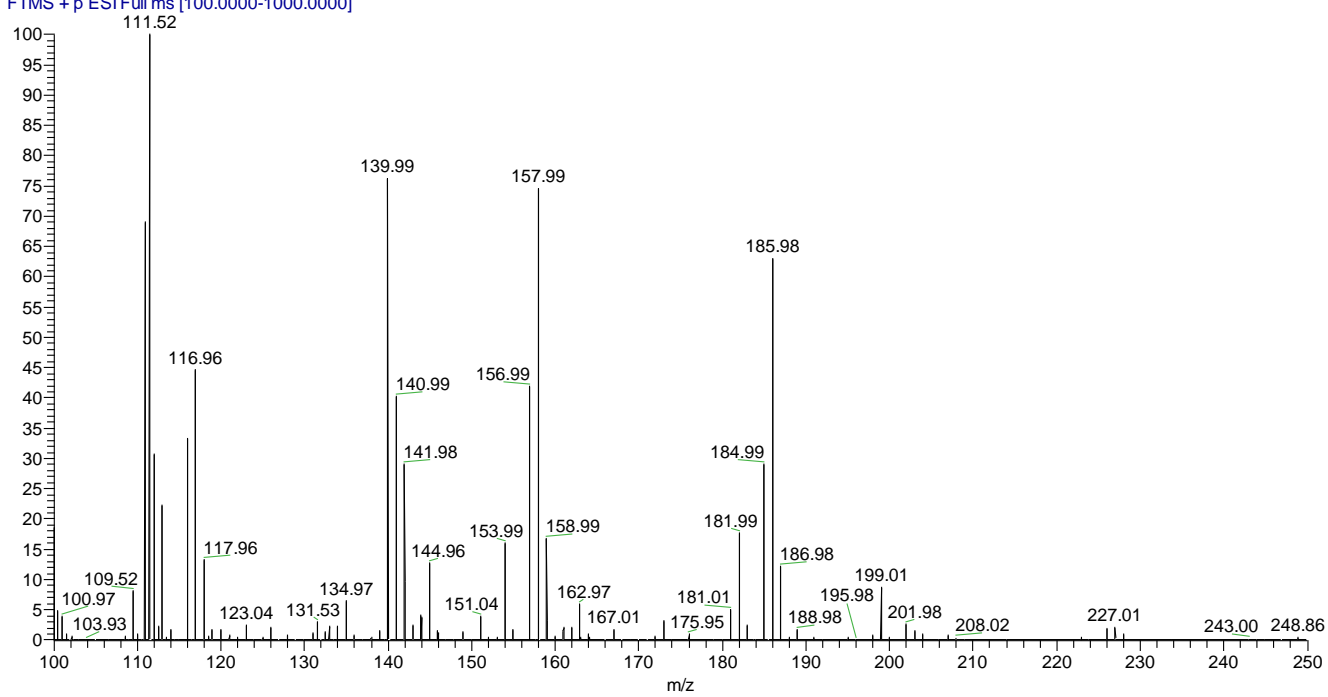

Figure S6. LC-MS spectra of 4-[2-imino-pyrimidine-1(2H)-yl]aniline (P1, m/z=187) during the reaction. 

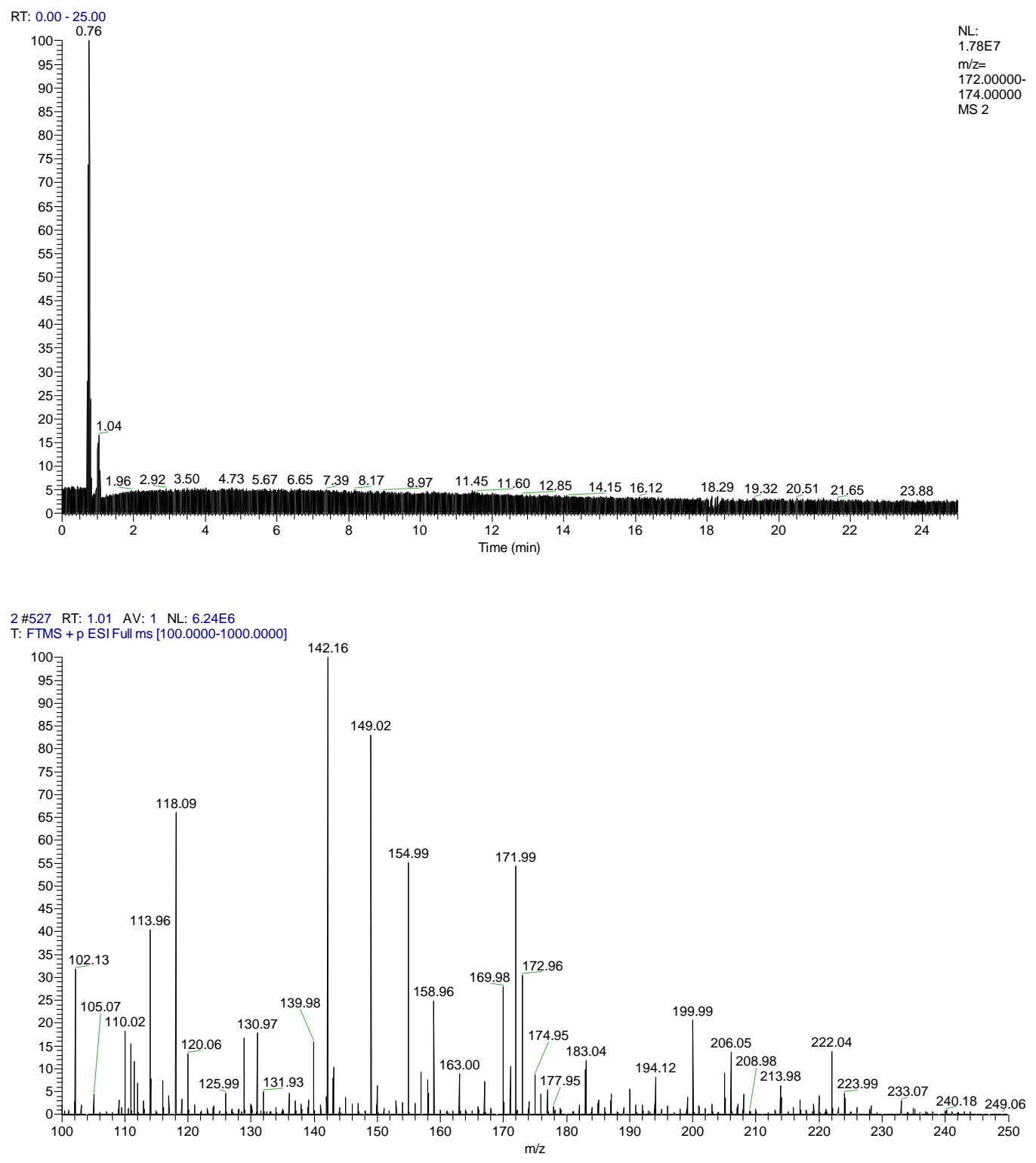

Figure S7. LC-MS spectra of sulfanilamide $(\mathrm{P} 2, \mathrm{~m} / \mathrm{z}=173)$ during the reaction. 


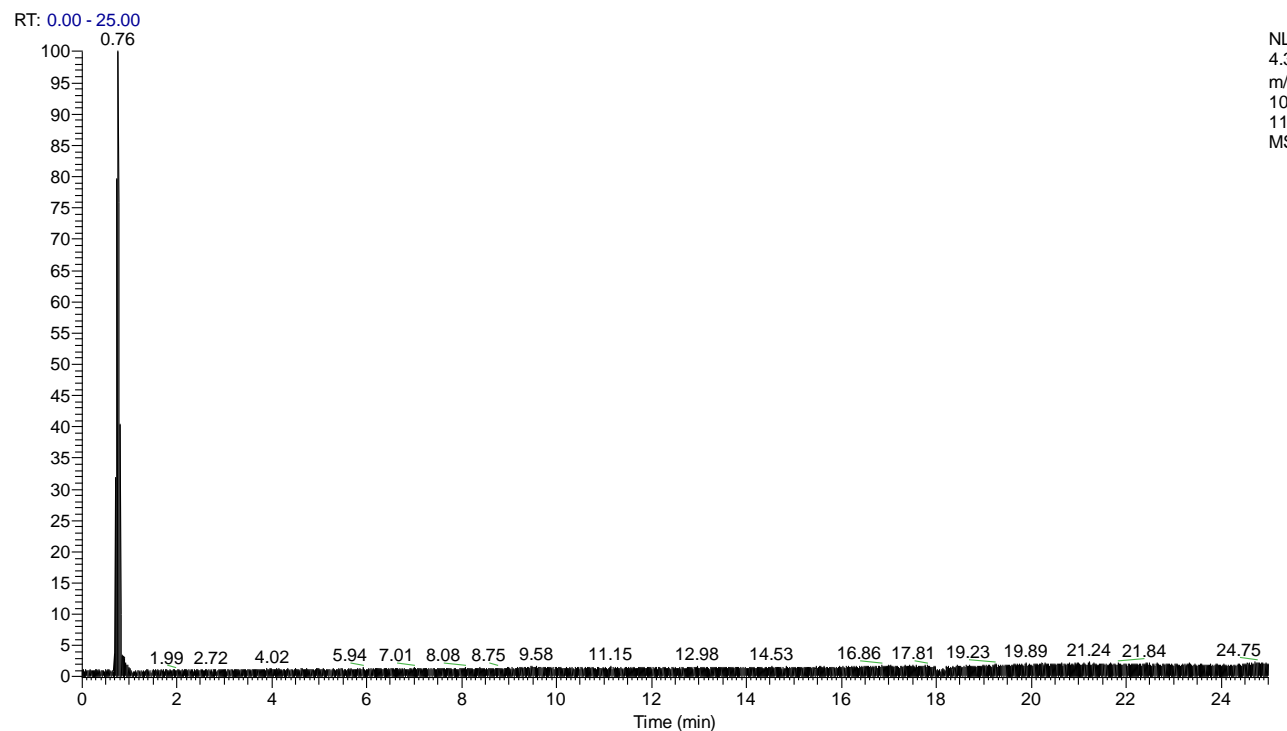

NL:

$\mathrm{m} / \mathrm{Z}=\mathrm{Z}$

MS 2

2 \#386 RT: 0.76 AV: $1 \quad \mathrm{NL}: 4.24 \mathrm{E} 6$

T: FTMS + p ESId Full ms2 151.0351@ncd28.00 [50.0

100 丰

95
90

80年

80 年

70 余

65 年

50 翠

50 事
40 事

40彗

25毒 $\quad 110.01$

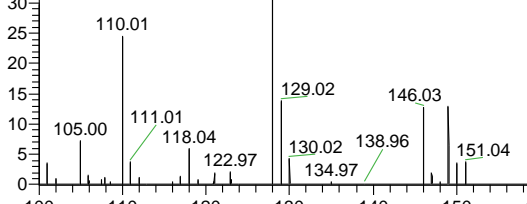

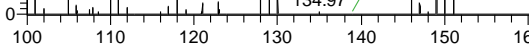

Figure S8. LC-MS spectra of 4-amino-phenol (P3, m/z=109) during the reaction. 

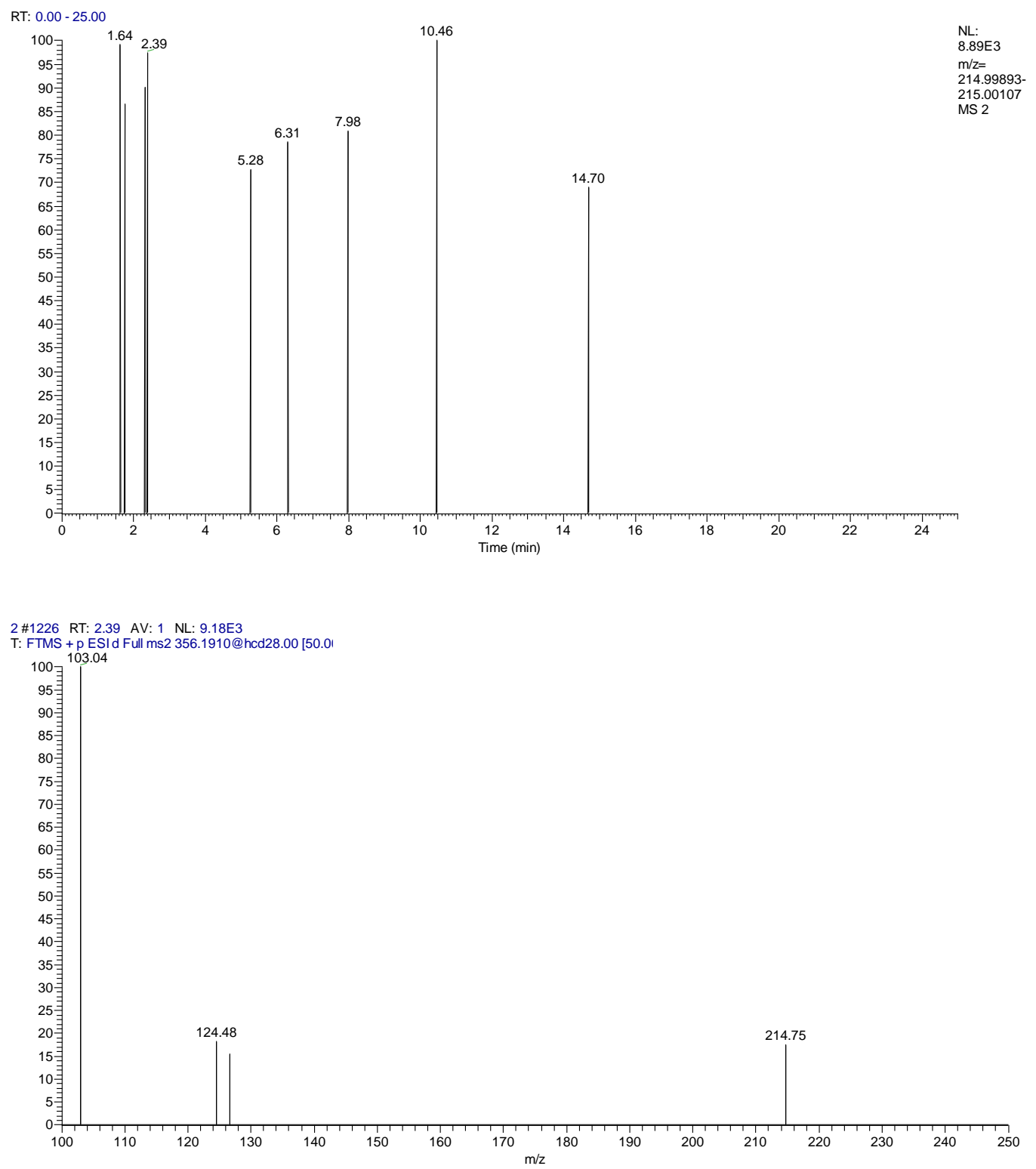

Figure S9. LC-MS spectra of sulfaguanidine $(\mathrm{P} 4, \mathrm{~m} / \mathrm{z}=215)$ during the reaction. 


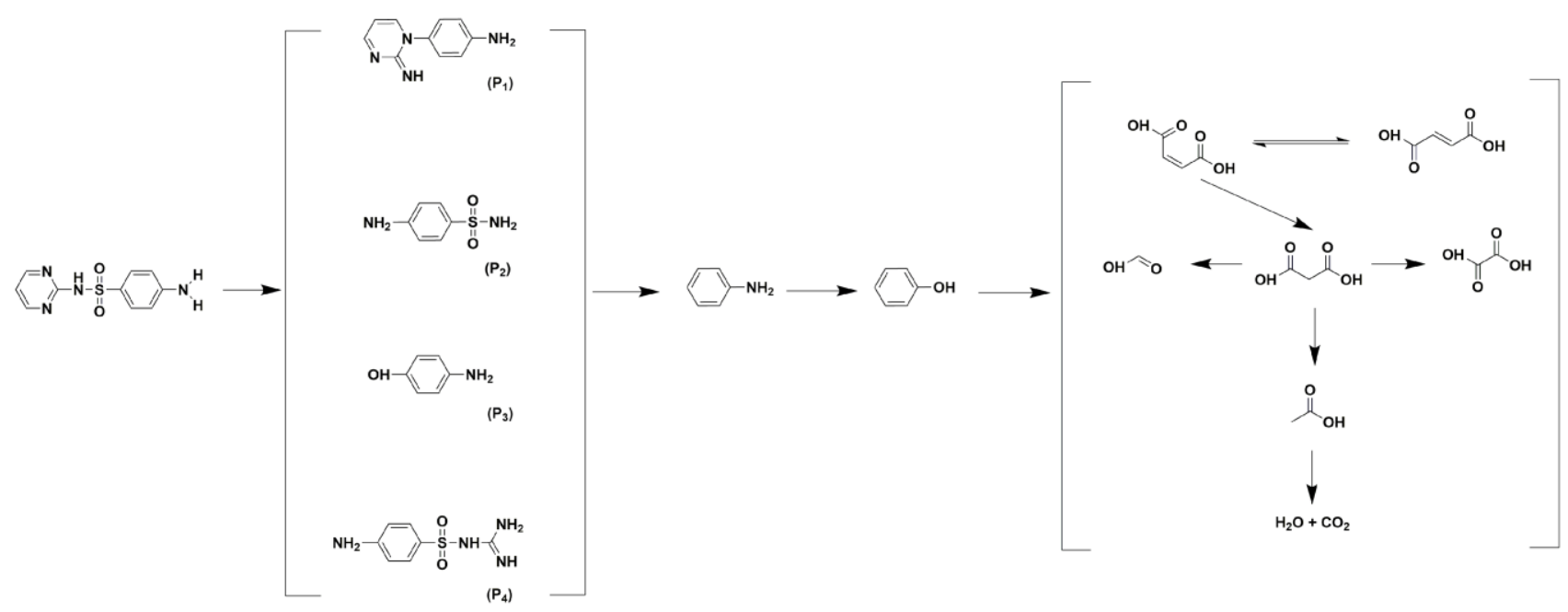

Figure S10. The possible degradation pathway of SDZ in SCNGs/PMS system based on LC-MS. 